\title{
Effects on cell proliferation and EPR spin trapping studies of Abnormal Savda Munziq and its chromatographic subfractions
}

\author{
Klaus Stolze ${ }^{1 *}$, Adiljan Ablimit ${ }^{2}$, Alois Strasser ${ }^{3}$ \\ From 16th Scientific Symposium of the Austrian Pharmacological Society (APHAR) \\ Vienna, Austria. 25-27 November 2010
}

\section{Background}

Abnormal Savda Munziq (ASMq) is a herbal preparation of traditional Uighur medicine for the prevention or treatment of a variety of diseases (e.g. bowel cancer, diabetes, hypertension and others).

\section{Methods}

In addition to the beneficial therapeutical effects of ASMq we also expected possible toxic effects and therefore focussed our in vitro investigations on inhibition of cell proliferation and also investigated its free radical scavenging as well as generating properties using electron spin resonance spectroscopy (ESR). ASMq was tested as aqueous solution as well as different subfractions obtained by sequential extraction with different solvents, i.e. $n$-hexane, methylene chloride, ethyl acetate, ethanol, methanol, methanol/water and finally water. Furthermore, the ethanolic fraction (containing the highest mass) was subsequently divided into chromatographic subfractions using semi-preparative HPLC. The whole aqueous and organic extracts as well as the ethanolic subfractions were subjected to determination of cell proliferative activity of murine hybridoma cells (YAC-1), which were treated with increasing concentrations of the respective extracts.

\section{Results}

We were able to demonstrate substantial effects of a set of subfractions of ASMq on cell proliferation with a significant correlation between decreasing proliferative activity and increasing lipophilic properties of the
ASMq-subfractions, also correlating to an increased retention time on semi-preparative $\mathrm{C}-18$ columns in our HPLC experiments. In our ESR experiments we observed significant antioxidant properties of the whole extract in spin trapping experiments using DEPMPO and a hydroxyl radical generating Fenton system. In addition, formation of a mixture of free radicals was observed under alkaline conditions suggesting the involvement of phenolic compounds. We were not able to identify the radicals involved; however, the polyphenolic compound gallic acid was identified by HPLC as a possible main active ingredient.

\section{Conclusions}

Our preliminary results show antioxidant properties of aqueous ASMq solutions. Free radicals are only detectable under alkaline conditions. Only the hydrophobic parts of ASMq inhibit cell proliferation.

\section{Author details}

'Department of Biomedical Sciences, Molecular Pharmacology and Toxicology, University of Veterinary Medicine Vienna, 1210 Viena, Austria. ${ }^{2}$ Xinjiang Medical University, Urumgi 830054, Xinjiang, China. ${ }^{3}$ Department of Biomedical Sciences, Institute of Physiology, University of Veterinary Medicine Vienna, 1210 Vienna, Austria.

Published: 16 November 2010

doi:10.1186/1471-2210-10-S1-A14

Cite this article as: Stolze et al:: Effects on cell proliferation and EPR spin trapping studies of Abnormal Savda Munziq and its chromatographic subfractions. BMC Pharmacology 2010 10(Suppl 1):A14.

\footnotetext{
* Correspondence: klaus.stolze@vetmeduni.ac.at

'Department of Biomedical Sciences, Molecular Pharmacology and

Toxicology, University of Veterinary Medicine Vienna, 1210 Viena, Austria

Full list of author information is available at the end of the article
} 